\title{
Measuring the growth rate of structure around cosmic voids
}

\author{
A. J. Hawken, D. Michelett, B. Granett, A. Iovino, L. Guzzo and + \\ VIPERS
}

${ }^{1}$ Osservatorio Astronomico di Brera, via E. Bianchi 46, 23807 Merate, Lc

\begin{abstract}
Using an algorithm based on searching for empty spheres we identified 245 voids in the VIMOS Public Extragalactic Redshift Survey (VIPERS). We show how by modelling the anisotropic void-galaxy cross correlation function we can probe the growth rate of structure.
\end{abstract}

Keywords. cosmology: large scale structure of the universe, observations, cosmological parameters, gravitation

\section{Introduction}

Different cosmological models, and different theories of gravity, predict that the large scale distribution of matter should be structured in subtly different ways. The light emitted from galaxies can be used as a proxy to trace this weblike structure. The cosmic web can be split into different component structures which show different properties, namely clusters, filaments, walls, and voids. Cosmic voids are the most underdense regions of the universe, and compose most its volume. They are also the most dark energy dominated environments and so are ideal places in which to study the vacuum energy and to search for signatures of modified gravity.

Galaxies used to trace voids are subject to motions apart from the Hubble flow. These motions contribute to the observed redshift of a galaxy and distort its apparent position in space. A galaxy in or close to the edge of a void is likely to be being evacuated away from the void centre, falling onto the surrounding structure (Padilla et al. 2005, Dubinski et al. 1993). These redshift space distortions (RSD) introduce an anisotropy to the voidgalaxy cross correlation function, $\xi_{v g}$.

Here we give an overview of the search for voids in VIPERS (Guzzo et al. 2013) and demonstrate how the anisotropy in the void-galaxy cross correlation function caused by linear redshift space distortions can be observed. Following this we propose that by fitting a model to the observed $\xi_{v g}$, which includes linear RSD, it is possible to extract a measurement of the growth rate of structure, $f(\Omega)$.

\section{The search for voids in VIPERS}

VIPERS aims to measure 100,000 redshifts for galaxies out to a redshift of $z \sim 1$ over an area of 24 square degrees. The current public data release contains $\sim 57000$ spectra. The survey is particularly narrow in declination which makes it difficult to use common void finding techniques such as the watershed algorithm (Platen et al. 2007, Neyrinck et al. 2008, Sutter et al. 2015). We therefore developed an algorithm which searches for voids using empty spheres, which is described in detail in Micheletti et al. 2014.

We searched for voids in a volume limited sample of galaxies with a redshift $0.55<$ $z<0.9$, from the VIPERS internal data release four. We first thinned the sample by removing galaxies with a third nearest neighbour distance more than $3.5 \sigma$ from the 
mean. Removing the most isolated galaxies increases the density contrast between high and low density regions. It also removes galaxies which have possibly been spuriously introduced to voids by non-linear RSD (Hamilton 1992). Furthermore, this provides us with a sample of the most isolated galaxies in VIPERS which can be analysed for galaxy evolution studies.

We then grow empty spheres on a fine regular grid. We limit ourselves to only searching for the most significant empty spheres. This is to avoid selecting spurious under densities generated by masking effects and in the hope that a sample of larger voids will avoid the void-in-cloud problem (Sheth \& van de Weygaert 2004). Empty spheres are deemed significant if they have a radius greater than $2.5 \sigma$ times the mean inter-galaxy separation, in practice this means that the empty spheres we are interested in have a radius $\gtrsim 15 \mathrm{Mpch}^{-1}$. Our voids are then defined as the regions connected by overlapping statistically significant spheres. The subset of statistically significant non-overlapping empty spheres are called maximal spheres. We identified 229 maximal spheres in 145 voids in the W1 field of VIPERS, and 159 maximal spheres in 100 voids in W4. The distinction between maximal spheres and voids is illustrated in figure 1 .

We measured the cross-correlation function between VIPERS galaxies (including those previously pruned out) and maximal spheres using the Davis and Peebles estimator (Davis \& Peebles 1983).

\section{A model for the void-galaxy cross correlation function}

Following Paz et al. 2013, we assume that the line of sight pairwise velocity dispersion approximately follows a Maxwell-Boltzman distribution,

$$
1+\xi_{v g}(\sigma, \pi)=\int \frac{\mathrm{d} w_{3}}{\sqrt{2 \pi} \sigma_{v}} \exp \left(-\frac{\left(w_{3}-v(r) \frac{r_{3}}{r}\right)^{2}}{2 \sigma_{v}}\right)\left[1+\xi_{v g}(r)\right],
$$

where $\xi_{v g}(\sigma, \pi)$ is the anisotropic cross correlation function, and $w_{3}$ is the line of sight component of the pairwise velocity. Because the densities in the vicinity of voids are small, the linear estimate for the relationship between the density and velocity fields remains valid,

$$
v(r) \approx-H r \Delta(r) \frac{f\left(\Omega_{m}\right)}{3} .
$$

The growth factor is commonly parameterised as $f\left(\Omega_{m}\right)=\Omega_{m}^{\gamma}$. In standard GR $\gamma \approx$ 0.55. Because the densities involved are very low, the gravitational dynamics of galaxies around voids remain in the linear regime. This should be particularly true for our void sample because our voids are relatively large and so are expected to be more linear. A correct description of the velocity field should also consider the impact of galaxy biasing. However, for now, we consider the bias to be constant and equal to unity.

The void galaxy cross-correlation function can be expressed in terms of the void density profile,

$$
\xi_{v g}(r)=\frac{1}{3 r^{2}} \frac{\mathrm{d}}{\mathrm{d} r}\left(r^{3} \Delta(r)\right) .
$$

There are several proposed functional forms for the void density profile in the literature. These can broadly be divided into two categories: phenomenological models which seek to fit the functional form of the void density profile (e.g. Hamaus et al. 2014, Paz et al. 2013), and theoretically motivated models (e.g. Fimelli et al. 2014). 


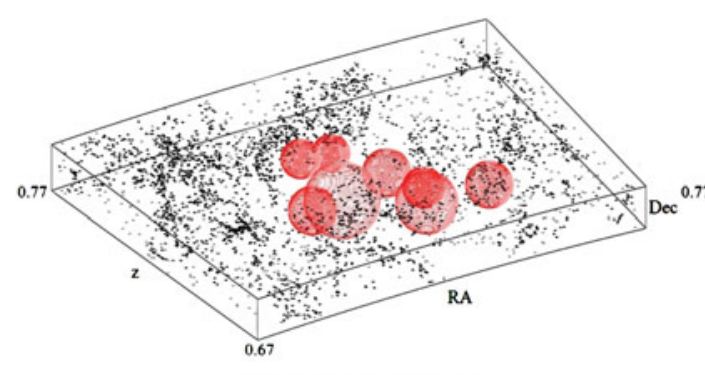

(a) Maximal spheres

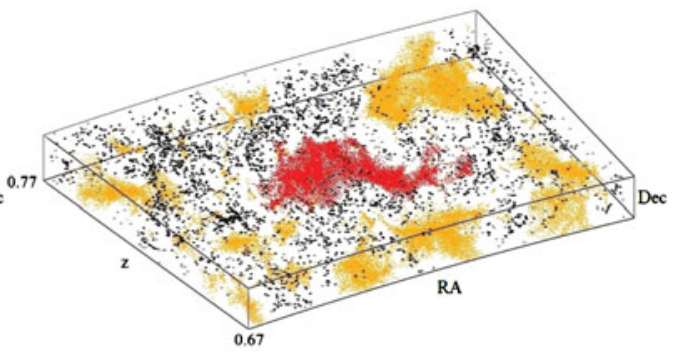

(b) Void region

Figure 1: We calculate the cross correlation between significant non overlapping spheres (a) and galaxies. By connecting together spheres which do overlap, our algorithm can be used to find void regions of arbitrary shape (b).

\section{Measuring the growth rate}

We ran our void finder on 57 mock VIPERS fields generated from the Multidark Nbody simulations with a fiducial cosmology of $\Omega_{m}=0.27, \Omega_{b}=0.0469, \Omega_{\Lambda}=0.73, H_{0}=$ $70 \mathrm{~km} \mathrm{~s}^{-1} \mathrm{Mpc}^{-1}, n_{s}=0.95$, and $\sigma_{8}=0.82$ (Prada et al. 2012, de la Torre et al. 2013). Using the publicly available Monte-Carlo Markov Chain analysis tool PYMC (Patil et al. 2010), we fitted a selection of void profile models to the mean of all the void-galaxy cross correlation functions measured in the mocks, keeping the growth rate fixed to the fiducial cosmology. In an attempt to re-extract the input cosmology we then varied $f(\Omega)$ keeping the other parameters fixed and calculated the most likely value for $f(\Omega)$. The likelihood that a given model is true is related to the $\chi^{2}$ in the usual way, $\mathcal{L}=\exp \left(-\chi^{2} / 2\right)$, where

$$
\chi^{2}=\sum_{i j} \frac{\mathcal{D}_{i j}-\mathcal{M}_{i j}}{\sigma_{i j}^{2}}
$$

where $\mathcal{D}_{i j}$ is the observed cross correlation function $\xi_{v g}(\sigma, \pi)$ in bin $i j, \mathcal{M}_{i j}$ is the value predicted by the model, and $\sigma_{i j}^{2}$ is the variance of the cross correlation function measured in bin $i j$. All the models we tested provide a considerably better fit to the mocks when they are normalised so that the central density of the void is fixed to the observed value $\delta_{c} \sim 0.85$. i.e. $\delta_{c}>-1$. Failing to account for the fact that the interiors of voids are not completely empty introduces a systematic bias which suppresses the measurement of the growth rate. Keeping the shape parameters fixed to the values measured in the mocks we then fitted the observed $\xi_{v g}(\sigma, \pi)$ as measured in VIPERS, varying $f(\Omega)$. Figure 2 shows the normalised likelihood for different values of $f(\Omega)$ obtained by fitting different models for $\xi_{v g}$. Also plotted are the conventional measurement of the growth rate from VIPERS, presented in de la Torre et al. 2013, and the expected value given a Planck like cosmology Ade et al. 2014. There is no significant tension between our most likely value of $f(\Omega)$ and that obtained from a conventional analysis of the VIPERS data.

\section{Conclusion}

We have demonstrated that it is possible to constrain the growth rate of structure by fitting a model to the anisotropic void-galaxy cross correlation function, as measured in VIPERS. However, the results are highly model dependent and are subject to unknown systematics which may be introducing biases. A paper exploring these issues is currently in preparation. 


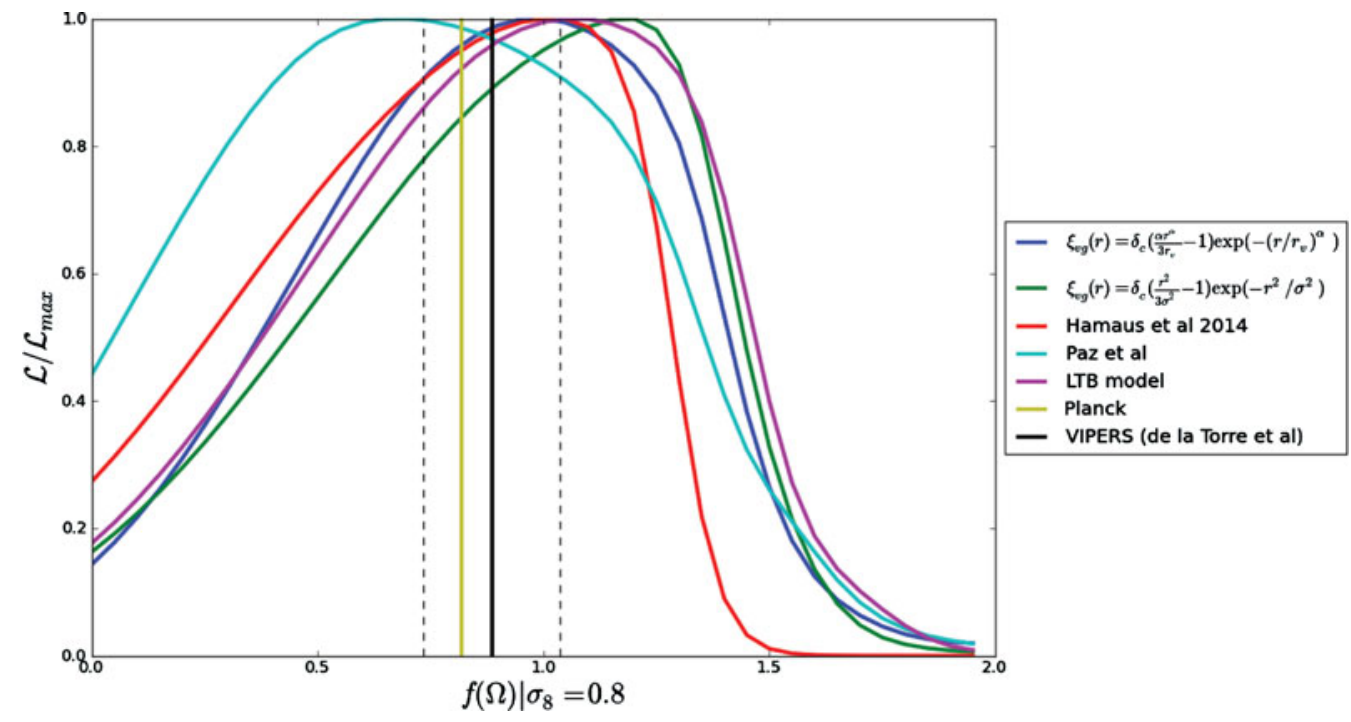

Figure 2: Normalised maximum likelihood for different values of the growth rate $f(\Omega)$, for various model density profiles given the VIPERS data. The solid and dashed black lines represent the measurement of the growth rate, with one-sigma error bars, from VIPERS using standard two-point galaxy clustering statistics. The yellow (pale) vertical line indicates the expected value for $f(\Omega)$ given the best fit Planck cosmological parameters.

\section{References}

Ade, P. A. R. \& The Planck Collaboration, 2014, A\&A, 571, A16

Aragon-Calvo, M. A. \& Szalay, A. S., 2013, MNRAS, 428, 4

Davis, M. \& Peebles, P. J. E. 1983, ApJ, 267, 465

de la Torre, S., Guzzo, L., Peacock, J. A., et al. 2013, AAP, 557, A54

Dubinski, J., da Costa, L. N., Goldwirth, D. S., Lecar, M., \& Piran, T. 1993, ApJ, 410

Finelli, F., Garcia-Bellido, J., Kovacs, A., Paci, F., \& Szapudi, I., 2014, ArXiv e-prints:1405.1555

Guzzo, L., The Vipers Team 2013, The Messenger, 151, 41

Hamaus, N., Sutter, P. M., \& Wandelt, B. D. 2014, PRL, 112

Hamilton, A. J. S. 1992, APJL, 385, L5

Kaiser, N. 1987, MNRAS, 227

Micheletti, D., Iovino, A., Hawken, A. J., Granett, B., \& The VIPERS team, 2014, A\&A 570, A106

Neyrinck, M. C. 2008, MNRAS, 386, 2101

Padilla, N. D., Ceccarelli, L., \& Lambas, D. G. 2005, MNRAS, 363, 977

Paranjape, A., Lam, T. Y., \& Sheth, R. K., 2012, MNRAS, 420, 1648

Patil, A., Huard, D., \& Fonnesbeck, C. J., 2010, Journal of Statistical Software, 35, 4

Paz, D., Lares, M., Ceccarelli, L., Padilla, N., \& Lambas, D. G. 2013, MNRAS, 436

Platen, E., van de Weygaert, R., \& Jones, B. J. T. 2007, MNRAS, 380, 551

Prada, F., Klypin, A. A., Cuesta, A. J., Betancort-Rijo, J. E., \& Primack, J., 2012 ,MNRAS, 423

Regos, E. \& Geller, M. J. 1991, ApJ, 377, 14

Sheth, R. K. \& van de Weygaert, R. 2004, MNRAS, 350, 517

Sutter, P. M., Lavaux, G., Hamaus, N., Pisani, A., Wandelt, B. D., Warren, M., VillaescusaNavarro, F., Zivick, P., Mao, Q., \& Thompson, B. B., 2015, Astronomy and Computing, 9 